\title{
Optimal Token Allocations in Solitaire Knock 'm Down
}

\author{
Arthur T. Benjamin \\ Department of Mathematics \\ Harvey Mudd College \\ Claremont, CA 91711 \\ benjamin@hmc.edu
}

\author{
Matthew T. Fluet \\ Department of Computer Science \\ Cornell University \\ Ithaca, NY 14853 \\ fluet@cs.cornell.edu
}

\author{
Mark L. Huber \\ Department of Statistics \\ Stanford University \\ Stanford, CA 94305 \\ mhuber@orie.cornell.edu
}

Submitted: February 28, 2000; Accepted: August 14, 2000

\begin{abstract}
In the game Knock 'm Down, tokens are placed in $N$ bins. At each step of the game, a bin is chosen at random according to a fixed probability distribution. If a token remains in that bin, it is removed. When all the tokens have been removed, the player is done. In the solitaire version of this game, the goal is to minimize the expected number of moves needed to remove all the tokens. Here we present necessary conditions on the number of tokens needed for each bin in an optimal solution, leading to an asymptotic solution.

MR Subject Classifications: primary: 91A60
\end{abstract}

\section{Introduction}

Knock 'm Down is a simple game to play that proves surprisingly difficult to analyze. At the beginning of the game, the player allocates $t$ tokens to $N$ bins. An allocation can be described by a vector $X=\left(x_{1}, x_{2}, \ldots, x_{N}\right)$ of non-negative integers, with $\sum x_{i}=t$. At each step of the game, a bin is chosen at random, with the probability of choosing bin $i$ fixed at $p_{i}>0$. If at least one token remains in bin $i$ when it is chosen, then one token is removed from the bin. The game continues until all of the tokens have been removed. 
In Solitaire Knock 'm Down, the goal is to remove all the tokens in the shortest time possible. Given $t$ tokens, $N$ bins, and a fixed probability vector $P=\left(p_{1}, p_{2}, \ldots, p_{N}\right)$, we will say that an initial token allocation $X^{*}=\left(x_{1}^{*}, x_{2}^{*}, \ldots, x_{N}^{*}\right)$ is optimal if it minimizes the expected time needed to remove all of the tokens.

Intuitively, one would expect the optimal token placement to resemble the shape of the probability histogram, so that roughly $t p_{i}$ tokens are placed in bin $i$. However, recursive calculations in [3] reveal that even when one can allocate tokens exactly proportional to the probabilities, this allocation is seldom optimal. For example, when $P=(1 / 3,2 / 3)$ and $t=9$, then $X^{*}=(2,7)$. When $t=1200$ with the same $P, X^{*}=(393,807)$. When $P=(.1, .2, .3, .4)$ and $t=10$, then $X^{*}=(0,2,3,5)$. In the original version of Knock 'm Down, described in [1], $t=12$ and $P$ is the "dice total" vector $\frac{1}{36}(1,2,3,4,5,6,5,4,3,2,1)$. Here $X^{*}=(0,0,1,2,2,3,2,1,1,0,0)$.

When $N=2$ and $P=(p, 1-p)$, it is shown in [2] that with $t$ tokens, the optimal allocation is $X^{*}=(m, t-m)$, where $m$ is the $p$ th percentile of the $\operatorname{Binomial}(t, p)$ distribution. As a consequence, $\lim _{t \rightarrow \infty} X^{*} / t=P$.

In this paper, we generalize these results to obtain necessary conditions for $X^{*}$ when $N>2$, and achieve the same asymptotic conclusion.

\section{The Token Adding Theorem and Consequences}

Given a probability distribution $P=\left(p_{1}, \ldots, p_{N}\right)$ and token allocation $X=\left(x_{1}, \ldots, x_{N}\right)$, we define the random variable $T_{X}$ to be the number of rolls needed to clear allocation $X$. Consider arbitrary bins $a$ and $b$, with $p_{a}<p_{b}$. Let $X_{a}$ and $X_{b}$ be the allocations obtained from $X$ by adding one token to bins $a$ and $b$ respectively. Our first theorem maintains that if $x_{a} / x_{b}$ is at least $p_{a} / p_{b}$ then $X_{a}$ has a greater expected clearing time than $X_{b}$. More precisely,

Theorem 1. The Token Adding Theorem. Let $X$ be a token allocation with $t$ tokens such that $p_{b} x_{a} \geq p_{a} x_{b}$, where $p_{a}<p_{b}$. Then $E\left[T_{X_{a}}\right]>E\left[T_{X_{b}}\right]$.

Proof. To clear allocation $X_{a}$, we must first clear suballocation $X$ and then clear the remaining token in bin $a$, if it has not already been removed. Thus $T_{X_{a}}=T_{X}+R_{a}$, where $R_{a}$ is the number of extra turns needed to clear the extra token in bin $a . R_{a}$ is either 0 or a geometric random variable with mean $1 / p_{a}$. Defining $R_{b}$ analogously, we obtain

$$
\begin{aligned}
E\left[T_{X_{a}}\right]-E\left[T_{X_{b}}\right] & =\left(E\left[T_{X}\right]+\frac{1}{p_{a}} \operatorname{Pr}\left(R_{a}>0\right)\right)-\left(E\left[T_{X}\right]+\frac{1}{p_{b}} \operatorname{Pr}\left(R_{b}>0\right)\right) \\
& =\frac{1}{p_{a}} \operatorname{Pr}\left(R_{a}>0\right)-\frac{1}{p_{b}} \operatorname{Pr}\left(R_{b}>0\right) .
\end{aligned}
$$

It remains to show that $\frac{1}{p_{a}} \operatorname{Pr}\left(R_{a}>0\right)>\frac{1}{p_{b}} \operatorname{Pr}\left(R_{b}>0\right)$.

Notice that $\operatorname{Pr}\left(R_{a}>0\right)$ is the probability that suballocation $X$ is cleared with bin $a$ chosen exactly $x_{a}$ times. Thus, we define $A_{n, m}$ to be the set of length $n$ sequences of bin choices such that $X$ is cleared on the $n^{\text {th }}$ turn with bin $a$ selected exactly $x_{a}$ times and 
bin $b$ selected $x_{b}+m$ times, where $m \geq 0 . \operatorname{Pr}\left(A_{n, m}\right)=\sum_{\alpha \in A_{n, m}} \operatorname{Pr}(\alpha)$, where $\operatorname{Pr}(\alpha)$ is the product of the probabilities of the values in the sequence. Note that $A_{n, m}$ may be empty for some values of $t, n$, and $m$. In particular, $A_{n, m}=\emptyset$ for $n<t$ and for $m>n-t$. Therefore,

$$
\operatorname{Pr}\left(R_{a}>0\right)=\sum_{n=t}^{\infty} \sum_{m=0}^{n-t} \operatorname{Pr}\left(A_{n, m}\right) .
$$

Similarly, let $B_{n, m}$ be the set of length $n$ sequences of bin choices such that $X$ is cleared on the $n^{\text {th }}$ turn with bin $b$ selected exactly $x_{b}$ times and bin $a$ selected $x_{a}+m$ times. Thus,

$$
\operatorname{Pr}\left(R_{b}>0\right)=\sum_{n=t}^{\infty} \sum_{m=0}^{n-t} \operatorname{Pr}\left(B_{n, m}\right) .
$$

Hence to prove our theorem, it suffices to prove the following

Lemma 1. Let $X$ be a token allocation with $t$ tokens and $N$ values such that $p_{b} x_{a} \geq p_{a} x_{b}$, where $p_{a}<p_{b}$. Then for $n \geq t>0$ and $0 \leq m \leq n-t, \frac{1}{p_{a}} \operatorname{Pr}\left(A_{n, m}\right) \geq \frac{1}{p_{b}} \operatorname{Pr}\left(B_{n, m}\right)$. Further, the inequality is strict in the case $n=t$ and $m=0$.

We begin with the case $n=t$ and $m=0$. Recalling the definitions of $A_{n, m}$ and $B_{n, m}$, we note that both $A_{t, 0}$ and $B_{t, 0}$ are the set of sequences of values of length $n$ such that $X$ is cleared on the $t^{t h}$ turn with exactly $x_{a} a$ 's and $x_{b} b$ 's. Hence $A_{t, 0}=B_{t, 0}$. Further, since $n=t$, neither $A_{t, 0}$ nor $B_{t, 0}$ is empty. Since, $p_{a}<p_{b}$, then $\frac{1}{p_{a}} \operatorname{Pr}\left(A_{t, 0}\right)>\frac{1}{p_{b}} \operatorname{Pr}\left(B_{t, 0}\right)$.

Next, consider the case $n>t$ and $m=0$. Again, we note that $A_{n, 0}=B_{n, 0}$. Thus, $\frac{1}{p_{a}} \operatorname{Pr}\left(A_{t, 0}\right) \geq \frac{1}{p_{b}} \operatorname{Pr}\left(B_{t, 0}\right)$. The inequality is strict except when $N=2$ and $m>t$, where $\operatorname{Pr}\left(A_{n, 0}\right)=\operatorname{Pr}\left(B_{n, 0}\right)=0$.

Finally, consider the case $n>t$ and $0<m \leq n-t$.

Let $X^{\prime}$ be the allocation of $t-x_{a}-x_{b}$ tokens with all $x_{a}+x_{b}$ tokens removed from bins $a$ and $b$ in allocation $X$. Let $T^{\prime}$ be the number of turns needed to clear $X^{\prime}$ using probability vector $P^{\prime}=\left(p_{1}^{\prime}, \ldots, p_{n}^{\prime}\right)$, where $p_{a}^{\prime}=p_{b}^{\prime}=0$, and $p_{i}^{\prime}=p_{i} /\left(1-p_{a}-p_{b}\right)$ for $i \neq a, b$. In other words, $T^{\prime}$ is the number of non- $a$ and non- $b$ turns needed to clear $X^{\prime}$.

We may write $\operatorname{Pr}\left(A_{n, m}\right)$ as the sum of two probabilities:

$$
\operatorname{Pr}\left(A_{n, m}\right)=\operatorname{Pr}\left(A_{1}\right)+\operatorname{Pr}\left(A_{2}\right),
$$

where $A_{1}$ is the event that $X$ is cleared on the $n^{t h}$ turn with exactly $x_{a} a$ 's and $x_{b}+m$ $b$ 's and the $n^{\text {th }}$ token removed is from bin $a . A_{2}$ is the event that $X$ is cleared on the $n^{\text {th }}$ turn with exactly $x_{a} a$ 's and $x_{b}+m b$ 's and the $n^{\text {th }}$ token removed is not in bin $a$ nor bin $b$. Note that since $m>0$, the $n^{\text {th }}$ token removed can not be from bin $b$.

Likewise, we may write $\operatorname{Pr}\left(B_{n, m}\right)$ as the sum of two probabilities:

$$
\operatorname{Pr}\left(B_{n, m}\right)=\operatorname{Pr}\left(B_{1}\right)+\operatorname{Pr}\left(B_{2}\right)
$$

where $B_{1}$ is the event that $X$ is cleared on the $n^{\text {th }}$ turn with exactly $x_{a}+m a$ 's and $x_{b}$ $b$ 's and the $n^{\text {th }}$ token removed is from bin $b . B_{2}$ is the event that $X$ is cleared on the $n^{\text {th }}$ 
turn with exactly $x_{a}+m a$ 's and $x_{b} b$ 's and the $n^{\text {th }}$ token removed is neither in bin $a$ nor bin $b$. Since $m>0$, the $n^{\text {th }}$ token removed can not be from bin $a$.

In order to show that $\frac{1}{p_{a}} \operatorname{Pr}\left(A_{n, m}\right) \geq \frac{1}{p_{b}} \operatorname{Pr}\left(B_{n, m}\right)$, we show that $\frac{1}{p_{a}} \operatorname{Pr}\left(A_{1}\right) \geq \frac{1}{p_{b}} \operatorname{Pr}\left(B_{1}\right)$ and $\frac{1}{p_{a}} \operatorname{Pr}\left(A_{2}\right) \geq \frac{1}{p_{b}} \operatorname{Pr}\left(B_{2}\right)$.

First, we show $\frac{1}{p_{a}} \operatorname{Pr}\left(A_{1}\right) \geq \frac{1}{p_{b}} \operatorname{Pr}\left(B_{1}\right)$. The inequality is obviously true when $\operatorname{Pr}\left(B_{1}\right)=$ 0 , (e.g., when $x_{b}=0$ ). Now when $\operatorname{Pr}\left(B_{1}\right) \neq 0$,

$$
\begin{aligned}
& \operatorname{Pr}\left(A_{1}\right)=\left(\begin{array}{c}
n-1 \\
x_{a}-1
\end{array}\right)\left(\begin{array}{c}
n-x_{a} \\
x_{b}+m
\end{array}\right) p_{a}^{x_{a}} p_{b}^{x_{b}+m} \operatorname{Pr}\left(T^{\prime} \leq n-x_{a}-x_{b}-m .\right) \\
& \operatorname{Pr}\left(B_{1}\right)=\left(\begin{array}{c}
n-1 \\
x_{b}-1
\end{array}\right)\left(\begin{array}{c}
n-x_{b} \\
x_{a}+m
\end{array}\right) p_{b}^{x_{b}} p_{a}^{x_{a}+m} \operatorname{Pr}\left(T^{\prime} \leq n-x_{a}-x_{b}-m .\right)
\end{aligned}
$$

Then,

$$
\begin{aligned}
\frac{\frac{1}{p_{a}} \operatorname{Pr}\left(A_{1}\right)}{\frac{1}{p_{b}} \operatorname{Pr}\left(B_{1}\right)} & =\frac{\frac{1}{p_{a}}\left(\begin{array}{c}
n-1 \\
x_{a}-1
\end{array}\right)\left(\begin{array}{c}
n-x_{a} \\
x_{b}+m
\end{array}\right) p_{a}^{x_{a}} p_{b}^{x_{b}+m} \operatorname{Pr}\left(T^{\prime} \leq n-x_{a}-x_{b}-m\right)}{\frac{1}{p_{b}}\left(\begin{array}{c}
n-1 \\
x_{b}-1
\end{array}\right)\left(\begin{array}{c}
n-x_{b} \\
x_{a}+m
\end{array}\right) p_{b}^{x_{b}} p_{a}^{x_{a}+m} \operatorname{Pr}\left(T^{\prime} \leq n-x_{a}-x_{b}-m\right)} \\
& =\frac{\frac{(n-1) !}{\left(x_{a}-1\right) !\left(x_{b}+m\right) !\left(n-x_{a}-x_{b}-m\right) !} p_{b}^{m+1}}{(n-1) !} \\
& =\left(\frac{p_{b}}{p_{a}}\right)^{m+1} \frac{\left(x_{b}-1\right) !\left(x_{a}+m\right) !}{\left(x_{a}-1\right) !\left(x_{b}+m\right) !} \\
& =\left(\frac{p_{b}}{p_{a}}\right)^{m+1} \frac{x_{a}\left(x_{a}+1\right) \cdots\left(x_{a}+m\right)}{x_{b}\left(x_{b}+1\right) \cdots\left(x_{b}+m\right)} \\
& =\prod_{k=0}^{m} \frac{p_{b}\left(x_{a}+k\right)}{p_{a}\left(x_{b}+k\right)} \\
& \geq 1 .
\end{aligned}
$$

The last step follows from our theorem's initial assumptions, since

$$
p_{b}\left(x_{a}+k\right)-p_{a}\left(x_{b}+k\right)=p_{b} x_{a}-p_{a} x_{b}+k\left(p_{b}-p_{a}\right) \geq 0 .
$$


By a similar calculation, when $\operatorname{Pr}\left(B_{2}\right) \neq 0$,

$$
\begin{aligned}
\frac{\frac{1}{p_{a}} \operatorname{Pr}\left(A_{2}\right)}{\frac{1}{p_{b}} \operatorname{Pr}\left(B_{2}\right)} & =\frac{\frac{1}{p_{a}}\left(\begin{array}{c}
n-1 \\
x_{a}
\end{array}\right)\left(\begin{array}{c}
n-x_{a}-1 \\
x_{b}+m
\end{array}\right) p_{a}^{x_{a}} p_{b}^{x_{b}+m} \operatorname{Pr}\left(T^{\prime}=n-x_{a}-x_{b}-m\right)}{\frac{1}{p_{b}}\left(\begin{array}{c}
n-1 \\
x_{b}
\end{array}\right)\left(\begin{array}{c}
n-x_{b}-1 \\
x_{a}+m
\end{array}\right) p_{b}^{x_{b}} p_{a}^{x_{a}+m} \operatorname{Pr}\left(T^{\prime}=n-x_{a}-x_{b}-m\right)} \\
& =\frac{\frac{(n-1) !}{x_{a} !\left(x_{b}+m\right) !\left(n-1-x_{a}-x_{b}-m\right) !}}{\frac{(n-1) !}{x_{b} !\left(x_{a}+m\right) !\left(n-1-x_{a}-x_{b}-m\right) !} p_{a}^{m+1}} \\
& =\left(\frac{p_{b}}{p_{a}}\right)^{m+1} \frac{x_{b} !\left(x_{a}+m\right) !}{x_{a} !\left(x_{b}+m\right) !} \\
& =\left(\frac{p_{b}}{p_{a}}\right)^{m+1} \frac{\left(x_{a}+1\right)\left(x_{a}+2\right) \cdots\left(x_{a}+m\right)}{\left(x_{b}+1\right)\left(x_{b}+2\right) \cdots\left(x_{b}+m\right)} \\
& =\frac{p_{b}}{p_{a}} \prod_{k=1}^{m} \frac{p_{b}\left(x_{a}+k\right)}{p_{a}\left(x_{b}+k\right)} \\
& \geq 1,
\end{aligned}
$$

as desired. The lemma and theorem are now established.

The Token Adding Theorem leads to practical necessary conditions for optimal token allocation. The first corollary establishes a relationship between the ratio of tokens in any two bins and their ratio of probabilities. Specifically,

Corollary 1. Let $X^{*}=\left(x_{1}^{*}, \ldots, x_{N}^{*}\right)$ be an optimal allocation of t tokens with probability vector $P=\left(p_{1}, \ldots, p_{n}\right)$. If $p_{a}<p_{b}$, then $p_{b}\left(x_{a}^{*}-1\right)<p_{a} x_{b}^{*}$.

Proof. Suppose $p_{b}\left(x_{a}^{*}-1\right) \geq p_{a} x_{b}^{*}$. Then by applying the Token Adding Theorem to the $t-1$-token allocation $X=\left(x_{1}^{*}, \ldots, x_{a}^{*}-1, \ldots, x_{N}^{*}\right)$, we see that $X^{*}$ can not be optimal since it has a higher average clearing time than allocation $\left(x_{1}^{*}, \ldots, x_{a}^{*}-1, \ldots, x_{b}^{*}+1, \ldots, x_{N}^{*}\right)$.

As an immediate corollary, we have the following intuitive result.

Corollary 2. Let $X^{*}=\left(x_{1}^{*}, \ldots, x_{N}^{*}\right)$ be an optimal allocation of $t$ tokens with probability vector $P=\left(p_{1}, \ldots, p_{n}\right)$. If $p_{a}<p_{b}$, then $x_{a}^{*} \leq x_{b}^{*}$.

By modifying the proof of the Token Adding Theorem, one can also obtain, as done in [3], the following two corollaries.

Corollary 3. Let $X^{*}=\left(x_{1}^{*}, \ldots, x_{N}^{*}\right)$ be an optimal allocation of t tokens with probability vector $P=\left(p_{1}, \ldots, p_{n}\right)$. If $p_{a}=p_{b}$, then $\left|x_{a}^{*}-x_{b}^{*}\right| \leq 1$.

Corollary 4. If the bins are arranged so that $P$ satisfies $p_{1} \geq p_{2} \geq \cdots \geq p_{N}$, then there exists an optimal allocation $X^{*}$ such that $x_{1}^{*} \geq x_{2}^{*} \geq \cdots \geq x_{N}^{*}$.

The four previous corollaries can be used to drastically cut down the number of candidates for optimal allocation. For instance, in the original game of Knock ' $m$ Down with $t=12$ tokens, the candidates for optimal allocation are reduced from 646,646 to 49 . 
However, some allocations, such as putting all tokens in the most probable bin, are not eliminated by the previous corollaries. In the next section, we obtain lower bounds for the optimal number of tokens in each bin, resulting in a natural allocation of tokens in the long run.

\section{Lower Bounds}

As in the previous section, given a configuration $X=\left(x_{1}, \ldots, x_{N}\right)$ where $\sum x_{i}=t$, we let $T_{X}$ denote the first time that all of the tokens have been removed. Then an optimal solution $X^{*}$ will satisfy $E\left[T_{X^{*}}\right] \leq E\left[T_{X}\right]$ for all configurations $X$.

Theorem 2. Let $X^{*}=\left(x_{1}^{*}, \ldots, x_{N}^{*}\right)$ be an optimal allocation of $t>0$ tokens, with probability vector $P=\left(p_{1}, \ldots, p_{N}\right)$. Then for $N \geq 2$, in each bin $i, x_{i}^{*}$ is at least as big as the $p_{i} /(N-1)$ percentile of the Binomial distribution with parameters $t$ and $p_{i}$.

Proof. When $N=2$, this is proved in [2]. For $N>2$, we shall prove something slightly stronger. The idea behind our proof is that in an optimal configuration, the probability of choosing from bin $i$ more than $x_{i}^{*}$ times before the end of the game should be small. For each bin $i$, let $\tau_{i}$ be the first time that bin $i$ is chosen $x_{i}^{*}+1$ times. If bin $i$ has been chosen more than $x_{i}^{*}$ times by the end of the game, then $\tau_{i}<T_{X^{*}}$. Otherwise, when bin $i$ is chosen exactly $x_{i}^{*}$ times at the end of the game, we set $\tau_{i}=\infty$. Note that $\tau_{i}$ cannot equal $T_{X^{*}}$ since the game cannot end on a "wasted" turn. Summarizing, $\tau_{i}>T_{X^{*}}$ if and only if bin $i$ is chosen exactly $x_{i}^{*}$ times at the end of the game. Our theorem is based on the following lemma.

Lemma 2. For $N>2$, let $X^{*}$ be an optimal allocation of t tokens. Then for each bin $i$,

$$
\operatorname{Pr}\left(\tau_{i}>T_{X^{*}}\right)>\frac{p_{i}}{N-1}
$$

Proof. We shall show the contrapositive. Let $X$ be a configuration with $\operatorname{Pr}\left(\tau_{i}>T_{X}\right) \leq$ $p_{i} /(N-1)$. We shall construct a new configuration $X^{\prime}$ with $E\left[T_{X^{\prime}}\right]<E\left[T_{X}\right]$.

Let $E_{j}$ be the event that a token in bin $j$ was the last one removed in the game. Consider the event $\tau_{i}<T_{X}$. When this occurs, all of the tokens in bin $i$ are gone before time $\tau_{i}$, but at least one token remains in some other bin. In particular, there exists at least one bin $j$ with $\operatorname{Pr}\left(E_{j} \mid \tau_{i}<T_{X}\right) \geq 1 /(N-1)$ and $x_{j} \geq 1$. Construct the new configuration $X^{\prime}$ by setting $x_{i}^{\prime}=x_{i}+1, x_{j}^{\prime}=x_{j}^{\prime}-1$ and $x_{k}^{\prime}=x_{k}$ for $k$ different from $i$ and $j$. In other words, $X^{\prime}$ is created from $X$ by transferring a token from bin $j$ to bin $i$.

Since $\tau_{i} \neq T_{X}$, we have,

$$
E\left[T_{X}\right]=E\left[T_{X} \mid \tau_{i}<T_{X}\right] \operatorname{Pr}\left(\tau_{i}<T_{X}\right)+E\left[T_{X} \mid \tau_{i}>T_{X}\right] \operatorname{Pr}\left(\tau_{i}>T_{X}\right),
$$

and

$$
E\left[T_{X^{\prime}}\right]=E\left[T_{X^{\prime}} \mid \tau_{i}<T_{X}\right] \operatorname{Pr}\left(\tau_{i}<T_{X}\right)+E\left[T_{X^{\prime}} \mid \tau_{i}>T_{X}\right] \operatorname{Pr}\left(\tau_{i}>T_{X}\right) .
$$

Now we show how these expectations relate to one another. 
Suppose that $\tau_{i}>T_{X}$. Then when all of the tokens of $X$ have been removed, one token still remains in bin $i$ under configuration $X^{\prime}$. The expected number of additional steps needed to remove this final token is $1 / p_{i}$. Therefore,

$$
E\left[T_{X^{\prime}} \mid \tau_{i}>T_{X}\right]=E\left[T_{X} \mid \tau_{i}>T_{X}\right]+\frac{1}{p_{i}} .
$$

Suppose that $\tau_{i}<T_{X}$. Then we have chosen bin $i$ at least $x_{i}+1=x_{i}^{\prime}$ times, and so no tokens remain in this bin. If any bin other than bin $j$ has the last token under $X$, then this will also be the last token under $X^{\prime}$ as well, and $T_{X}=T_{X^{\prime}}$. On the other hand, if $j$ is the last token remaining under $X$, at this point there are no tokens left under $X^{\prime}$, so $T_{X^{\prime}}<T_{X}$. The expected time to remove this final token from bin $j$ is $1 / p_{j}$, so altogether

$$
\begin{aligned}
E\left[T_{X^{\prime}} \mid \tau_{i}<T_{X}\right]= & E\left[T_{X^{\prime}} \mid \tau_{i}<T_{X}, E_{j}\right] \operatorname{Pr}\left(E_{j} \mid \tau_{i}<T_{X}\right) \\
& +E\left[T_{X^{\prime}} \mid \tau_{i}<T_{X}, \bar{E}_{j}\right] \operatorname{Pr}\left(\bar{E}_{j} \mid \tau_{i}<T_{X}\right) \\
= & \left(E\left[T_{X} \mid \tau_{i}<T_{X}, E_{j}\right]-\frac{1}{p_{j}}\right) \operatorname{Pr}\left(E_{j} \mid \tau_{i}<T_{X}\right) \\
& +E\left[T_{X} \mid \tau_{i}<T_{X}, \bar{E}_{j}\right] \operatorname{Pr}\left(\bar{E}_{j} \mid \tau_{i}<T_{X}\right) \\
= & E\left[T_{X} \mid \tau_{i}<T_{X}\right]-\frac{1}{p_{j}} \operatorname{Pr}\left(E_{j} \mid \tau_{i}<T_{X}\right) \\
\leq & E\left[T_{X} \mid \tau_{i}<T_{X}\right]-\frac{1}{(N-1) p_{j}} \\
< & E\left[T_{X} \mid \tau_{i}<T_{X}\right]-\frac{1}{(N-1)\left(1-p_{i}\right)}
\end{aligned}
$$

Putting it all together, we have that

$$
\begin{aligned}
E\left[T_{X^{\prime}}\right]< & \left(E\left[T_{X} \mid \tau_{i}>T_{X}\right]+\frac{1}{p_{i}}\right) \operatorname{Pr}\left(\tau_{i}>T_{X}\right) \\
& +\left(E\left[T_{X} \mid \tau_{i}<T_{X}\right]-\frac{1}{(N-1)\left(1-p_{i}\right)}\right) \operatorname{Pr}\left(\tau_{i}<T_{X}\right) \\
= & E\left[T_{X}\right]+\operatorname{Pr}\left(\tau_{i}>T_{X}\right)\left(\frac{1}{p_{i}}+\frac{1}{(N-1)\left(1-p_{i}\right)}\right)-\frac{1}{(N-1)\left(1-p_{i}\right)} .
\end{aligned}
$$

Therefore, since $\operatorname{Pr}\left(\tau_{i}>T_{X}\right) \leq \frac{p_{i}}{N-1}$,

$$
\begin{aligned}
E\left[T_{X^{\prime}}\right]-E\left[T_{X}\right] & <\frac{1}{N-1}+\frac{p_{i}}{(N-1)^{2}\left(1-p_{i}\right)}-\frac{1}{(N-1)\left(1-p_{i}\right)} \\
& =\frac{(N-1)\left(1-p_{i}\right)+p_{i}-(N-1)}{(N-1)^{2}\left(1-p_{i}\right)} \\
& =\frac{p_{i}(2-N)}{(N-1)^{2}\left(1-p_{i}\right)} \\
& <0 .
\end{aligned}
$$

Thus, $E\left[T_{X^{\prime}}\right]<E\left[T_{X}\right]$, and $X$ is not an optimal solution, establishing our lemma. 
To complete the proof of Theorem 2, suppose that $x_{i}^{*}$ was less than the $p_{i} /(N-1)$ percentile of the Binomial distribution with parameters $t$ and $p_{i}$. Then since $T_{X^{*}} \geq t$,

$$
\begin{aligned}
\operatorname{Pr}\left(\tau_{i}>T_{X^{*}}\right) & \leq \operatorname{Pr}\left(\tau_{i}>t\right) \\
& =\operatorname{Pr}\left(\operatorname{Bin} i \text { is chosen at most } x_{i}^{*} \text { times among the first } t \text { turns }\right) \\
& <p_{i} /(N-1),
\end{aligned}
$$

contradicting our lemma.

Because $p_{i} /(N-1)$ is a constant, and the binomial distribution concentrates near $t p_{i}$ for large $t$, we have as an immediate corollary,

Corollary 5. Let $X^{*}=\left(x_{1}^{*}, \ldots, x_{N}^{*}\right)$ be an optimal allocation of t tokens with probability vector $P=\left(p_{1}, \ldots, p_{n}\right)$. Then $\lim _{t \rightarrow \infty} X^{*} / t=P$.

\section{Acknowledgment}

We thank Professor Janet Myhre and The Reed Institute for Applied Mathematics for support of this research.

\section{References}

[1] A.T. Benjamin and M.T. Fluet, The Best Way to Knock 'm Down, The UMAP Journal, vol 20.1, 11-20, 1999.

[2] A.T. Benjamin and M.T. Fluet, What's Best?, The American Mathematical Monthly, vol 107, No. 6, 560-562, 2000.

[3] M.T. Fluet, Searching for Optimal Strategies in Knock 'm Down, senior thesis, Harvey Mudd College, Claremont, CA, 1999. 